\title{
A snake-based approach to accurate determination of both contact points and contact angles
}

\author{
A.F. Stalder ${ }^{\text {a }}$, G. Kulik ${ }^{\text {b }}$, D. Sage ${ }^{\text {a, }}$, L. Barbieri ${ }^{\text {b }}$, P. Hoffmann ${ }^{\text {b }}$ \\ ${ }^{a}$ Biomedical Imaging Group, Ecole Polytechnique Fédérale de Lausanne (EPFL), CH-1015 Lausanne, Switzerland \\ ${ }^{\mathrm{b}}$ Nanostructuring Research Group, Ecole Polytechnique Fédérale de Lausanne (EPFL), CH-1015 Lausanne, Switzerland
}

Received 14 July 2005; received in revised form 6 March 2006; accepted 7 March 2006

Available online 16 March 2006

\begin{abstract}
We present a new method based on B-spline snakes (active contours) for measuring high-accuracy contact angles. In this approach, we avoid making physical assumptions by defining the contour of the drop as a versatile B-spline curve. When useful, we extend this curve by mirror symmetry so that we can take advantage of the reflection of the drop onto the substrate to detect the position of the contact points. To keep a wide range of applicability, we refrain from discretizing the contour of the drop, and we choose to optimize an advanced image-energy term to drive the evolution of the curve. This term has directional gradient and region-based components; additionally, another term-an internal energy-is responsible for the snake elasticity and constrains the parameterization of the spline. While preserving precision at the contact points, we limit the computational complexity by constraining a non-uniform repartition of the control points. The elasticity property of the snake links the local nature of the contact angle to the global contour of the drop. A global knowledge of the drop contour allows us to use the reflection of the drop on the substrate to automatically and precisely detect a line of contact points (vertical position and tilt). We apply cubic-spline interpolation over the image of the drop; then, the evolution procedure takes part in this continuous domain to avoid the inaccuracies introduced by pixelization and discretization.

We have programmed our method as a Java software and we make it freely available [A.F. Stalder, DropSnake, Biomedical Imaging Group, EPFL, [ON LINE] visited 2005. http://bigwww.epfl.ch/demo/dropanalysis]. Our experiments result in good accuracy thanks to our high-quality image-interpolation model, while they show applicability to a variety of images thanks to our advanced image-energy term.
\end{abstract}

(C) 2006 Elsevier B.V. All rights reserved.

Keywords: Image processing; Drop shape analysis; Snake (Active contour); Contact angle; Contact point

\section{Introduction}

Wetting phenomena have been studied scientifically during the past 200 years with strongly varying interest. An excellent overview appeared recently in literature [2]. Thomas Young introduced in 1805 a simple equation that equilibrates the forces at the contact point of a liquid drop on a solid surface [3].Thomas Young's equation is

$\gamma_{l, g} \cos \theta=\gamma_{l, g}-\gamma_{s, l}$,

where $\gamma$ denote the excess free energy per unit area of the interface indicated by its indices $g, l$, and $s$, corresponding to the

\footnotetext{
* Corresponding author. Tel.: +41 2169351 89; fax: +41 216933701.

E-mail address: daniel.sage@epfl.ch (D. Sage).
}

gas, liquid, and solid phases, respectively. This expression is called Young's equation and remains to this day the most-used expression in the study of surface wetting. The well-known and tabulated values for the liquid/gas-excess free energy $\gamma_{l, g}$ correspond to the surface tension of the liquid with its vapor. Of even more relevance to this paper is the contact angle $\theta$, which is the other experimentally easily accessible factor in Young's equation.

In recent years, there has been an incredible renaissance of wetting studies, starting with the discovery of the lotus effect [4] and leaving many questions open [5]. The measurements of static angles of contact are generally considered to be precise to $\pm 3^{\circ}$, this residual variation being due to experimental conditions and operator non-reproducibility. The latter can be improved by less-subjective image-processing algorithms, as we shall discuss in this paper. 
The study of the dynamics of liquid drops impinging onto surfaces [6] has recently been shown to help improving the fabrication of micro-arrays [7]. (Micro-Arrays are recognized as key devices in present and future biomedical research.) Another way of studying dynamic aspects of wetting is by tilting the substrate or by increasing or decreasing the volume of the drop. Such studies typically measure the advancing and receding contact angle and reveal its hysteresis; they are carried out on time scales of several seconds, if not minutes. During that long duration, liquid molecules might spread on the not-yet wetted surface, and are considered as being the cause for the existence of a wetting hysteresis [8]. Meanwhile, fast dynamic measurements of liquid drops impinging onto surfaces can be carried out by high-speed cameras. Since those show that the drops deviate strongly from being spherical, the determination of the contact angles is more difficult, but a single movie of the interaction of the drop onto the solid surface is sufficient to measure the dynamic angles of the liquids and to permit the determination of the advancing and receding angles, at time scales coming close to the limit of supersonic monolayer coverage.

To better understand the temporal evolution of the contact angles, it would be very useful to determine the latter at sufficient high speed and precision. We therefore need a rapid and robust method to determine the angle of contact for the systematic study of wetting properties on micro- and nano-structured surfaces with homogeneous surface chemistry.

Nowadays, the technique of the sessile drop is the mostwidely used method to measure the contact angle. Due to the difficulties encountered to accurately estimate the contact angle, the domain has had a long-standing development. Direct measurement using goniometer on telescope, protractor on pictures (or its computer-based equivalent) are still widely used. The major drawback of these methods are the subjectivity due to the operator action. Therefore, it is often preferred to measure this angle indirectly. This can be done using either a global model of the drop or a local model at its contact points.

By approximating the contour as a sphere, a few points from the profile of a drop is all it takes to easily obtain a contact angle. But, in many situations, neglecting gravity and using the spherical assumptions is inaccurate because the sessile-drop method is most often used in the presence of gravity (or of any other field that is constant and perpendicular to the surface). In such conditions, if the surface is horizontal and homogeneous, one may consider the drop to be axisymmetric.

The ADSA method, which stands for axisymmetric dropshape analysis $[9,10]$, has been thoroughly investigated and its limitations are well-known [10]. It requires solving a Laplace equation, often by numerical integration. After discretization of the contour of a drop on an image, it searches for the best Laplace profile that corresponds to this contour. One may then obtain an accurate contact angle as well as a value for the capillary constant. However, drops are rarely perfectly axisymmetric. When characterizing surfaces, the difference in contact angle on different sides of a drop is a precious indicator of surface heterogeneities. Therefore, the use of axisymmetric models is inherently limited because the axisymmetricity hypothesis is not fulfilled in many cases.
Since global models have a limited validity, more local models may be preferable. The polynomial-fitting approach is one of them, where a certain number of coordinates from the contour of the drop near the contact points are extracted and fitted to a polynomial of a certain degree. Unfortunately, the resulting contact angle depends highly on the polynomial degree and on the number of coordinates points [11]. Despite these delicate issues, polynomial fitting remains the method of choice when considering non-axisymmetric drops, as shown in several comparative studies $[11,12]$.

In this paper, we propose an alternative method that retains the better aspects of both local and global models. Our new approach, based on snakes, reconciles the fact that the shape of a drop is global, with the fact that its angles of contact are local. Conversely, a snake may reveal local contact angles while keeping a global shape, because it depends on elasticity constraint which maintain it as a global entity, even though the forces influencing it are of limited range [13].

Although the position of the contact points is of critical importance when measuring an angle of contact, up to now it has been mainly measured by hand because, when using the sessiledrop method for characterizing surfaces, the position of the line of contact may change from one experiment to the next due to surface thickness or misalignments. In this paper, thanks to our global knowledge of the shape of the drop, we have been able to automatize the detection of the interface between the drop and the substrate it rests on. This interface may not be detected directly as it appears blurry and curved on the image. However, the reflection of the drop from the surface allows us to determine the position of the contact points. Finally, we use the global shape of the snake to accurately detect the profile of the drop at the points of contact.

In other words, the snake may be equivalent to the polynomial fitting approach for the determination of the contact angle, while still using global knowledge of the drop to accurately determine position of the drop and contact points. This is why it is the perfect tool to analyze images of entire drops profiles with apparent drop reflection.

Traditionally, the analysis of drop shapes was based on discrete contours which were obtained using simple edge detectors such as Sobel [14] or using more advanced methods such as Jensen-Shannon divergence-based methods [15]. Depending on the image characteristics and on the segmentation method, the edges of the drop were detected with various degrees of success. In some cases, especially when the image is not sharp, such discrete approaches fail [16].

A recent variant of ADSA, called theoretical image fitting analysis (TIFA), deals with a continuously defined drop contour. It uses a gradient-based error function and is consequently able to handle smooth images (e.g., captive bubbles) for which ADSA fails [16]. First, a theoretical gradient image is built using a numerical solution to the Laplace equation; then, the error function is defined as the sum of the square of the difference between an experimental gradient image and the theoretical one. In this approach, the contour is no more discretized, and the optimization takes into account continuously defined gradient values. This can extend the analysis of drop shapes to domains 
where the approaches based on edge detectors would fail because the images of the drops are too smooth.

To base segmentation on image energies is a very active research domain. For example, it has been suggested that exploiting the direction of the gradient could be useful in building some form of gradient-based image energies [18]. (Indeed, the direction of the gradient is certainly relevant when it comes to measuring angles.) Meanwhile, region-based energies are no less interesting, in part because they are known to be very robust. This is particularly true in the context of the analysis of drop shapes, since measurements are realized most of the time in dedicated environments and result in images with well-controlled pixel intensities. Following advances in this domain, we suggest to use a unified image energy that takes into account both a directional gradient energy and a region-based energy [19].

Cubic-spline interpolation has already been used to sample the contour of the drop [14]; in that approach, the role of interpolation is to allow for a sub-pixel refinement of the contour. In another contribution [17], horizontal spline interpolation on the gradient image has been used in the context of gradient energies. In this paper, we too choose to consider that the image pixels are the samples of a continuously defined image; but then, we pay attention to ensure that all the subsequent operations we apply are consistent with this model, in accordance with sampling theory. We propose to apply cubic-spline image interpolation to obtain sub-pixel resolution, and, accordingly, to consider an image energy based on a continuously defined contour of the drop. Due to its good properties, a spline-based gradient operator may also be used [20].

\section{Spline-based representation of the drop contours}

Parametric spline curves are very common in computer graphics. A spline of order $d^{1}$ is a piecewise-polynomial function consisting of concatenated polynomial segments of order $d$ that are joined at breakpoints [21]. Such parametric curves are attractive because of their capability to represent simple shapes with just a few spans. In the particular form of splines called B-splines, the spline function is obtained as a sum of a finite number of basis functions. As each basis function has a finite support, this is a computationally efficient way of representing splines.

\subsection{Parametric spline representation}

Because of their minimum-curvature property, computational effectiveness, and simplicity, cubic B-splines have been chosen as interpolating basis function. Indeed, as B-spline produce smooth curves, drops present as well only continuous regular contours. In addition, as the optimal solution for a curvatureconstrained snake is a curvilinear cubic spline [22], a B-spline snake may ideally represent the contour of resting drop in the absence of external forces. If a contour deviate from the minimum curvature property, the knots would deviate from their ideal

\footnotetext{
${ }^{1}$ Note that $d=n+1$, where $n$ is the degree of the spline or polynomial.
}

minimum curvature position. Eventually, if a contour deviate strongly from the minimum curvature, non-curvilinear splines are suggested (Section 4.1). A correct representation of the contour of a drop may thus be expected using only a limited number of B-spline segments.

A cubic-spline parametric open curve in the $x-y$ plane may be described

$\forall t \in[0, M]:\left\{\begin{array}{l}x(t)=\sum_{k=-1}^{M+1} c_{x, k} \beta^{3}(t-k) \\ y(t)=\sum_{k=-1}^{M+1} c_{y, k} \beta^{3}(t-k)\end{array}\right.$

and by its derivatives

$\forall t \in[0, M]:\left\{\begin{array}{l}x^{\prime}(t)=\sum_{k=-1}^{M+1} c_{x, k} D \beta^{3}(t-k) \\ y^{\prime}(t)=\sum_{k=-1}^{M+1} c_{y, k} D \beta^{3}(t-k),\end{array}\right.$

where $\beta^{3}$ is the cubic $\mathrm{B}$-spline, where $\left(c_{x, k}, c_{y, k}\right)$ are the coordinates of the $k$ th control point among $M$ control points $c_{k}$, and where $D$ is the differential operator $\frac{\mathrm{d}}{\mathrm{d} t}$.

Note that a cubic spline does not interpolate its control points. Using IIR filter, one may obtain interpolating equivalents of control points: the knots, or nodes [22].

\subsection{Boundary conditions}

A cubic spline at regular breakpoints has a continuity $C^{2}$ : the first and second derivatives are continuous. However, the curve of the drop must have a discontinuity of its first derivative in order to represent angles. Consequently, border conditions must be applied to the spline at the contact points. In order to achieve that, triple control points may be used [21]. However, such an approach would introduce straight segments and a spurious parameterization [23].

Phantom vertices are additional control points at boundaries. Phantom vertices constrain the spline to interpolate the boundary control points as well as to have its tangent at these boundary points passing through the phantom point and its source [23]. In order to achieve such a goal, a phantom point added at one end of the spline must be a prolongation of the spline by symmetry through the last control point. We illustrate in Fig. 1 an endvertex interpolation that uses phantoms.

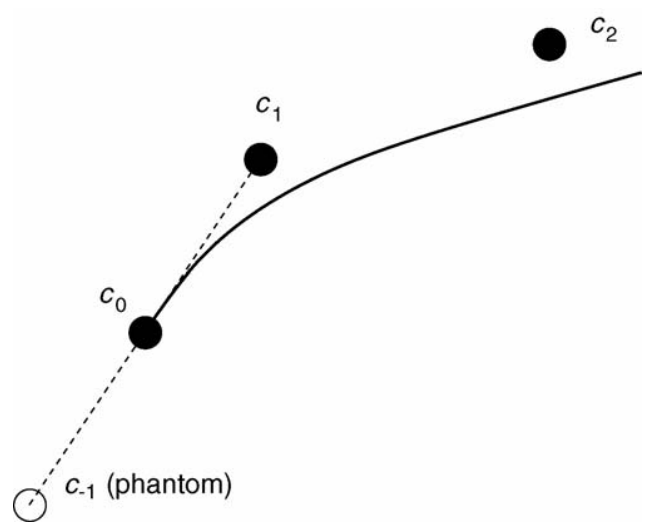

Fig. 1. Illustration of end-vertex interpolation via phantom vertices. The phantom control point $c_{-1}$ is obtained by a symmetry of $c_{1}$ through $c_{0}$. 


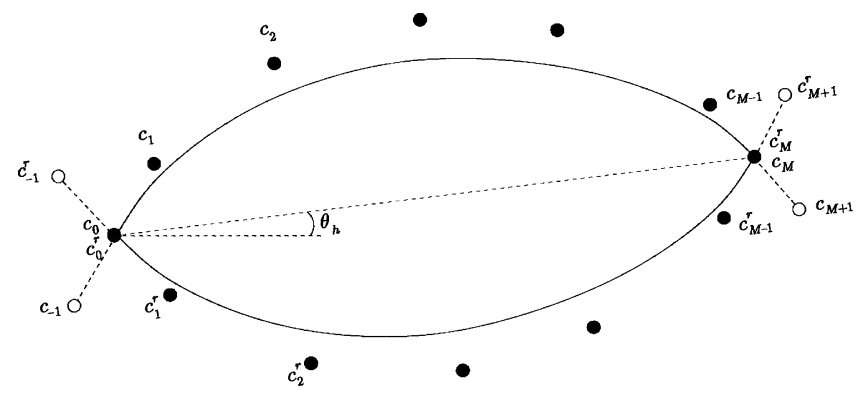

Fig. 2. Illustration of control-points parameterization. The native contour of the drop is represented with the spline defined by the $c_{k}$ s. The reflected contour of the drop is represented with the spline defined by the $c_{k}^{\mathrm{r}} \mathrm{s}$. The mirror symmetry line is defined by the position of $c_{0}=c_{0}^{\mathrm{r}}$ and $c_{M}=c_{M}^{\mathrm{r}}$. It exhibits a tilt-angle $\theta_{\mathrm{h}}$ with the horizontal.

\subsection{Symmetric model of drops using drop reflection}

When the reflection of the drop on the substrate is present in the image, the contact points may be detected automatically. In order to do so, the drop spline should be extended by mirror symmetry at its boundary control points. The contour of the drop is now defined by one spline for its non-reflected contour, and by one spline for its reflected contour. The non-reflected drop profile is defined by

$\left\{\begin{array}{l}x_{\text {sup }}(t)=\sum_{k=-1}^{M+1} c_{x, k} \beta^{3}(t-k) \\ y_{\text {sup }}(t)=\sum_{k=-1}^{M+1} c_{y, k} \beta^{3}(t-k),\end{array}\right.$

where $\left(c_{x, k}, c_{y, k}\right)$ is the coordinate of the control point for the non-reflected profile spline, and where $\left(c_{x,-1}, c_{y,-1}\right)$ and $\left(c_{x, M+1}, c_{y, M+1}\right)$ are the phantoms edge control points coordinates. The reflected profile of the drop is defined by a symmetry of the control points from the original profile, as in

$\left\{\begin{array}{l}x_{\text {inf }}(t)=\sum_{k=-1}^{M+1} c_{x, k}^{\mathrm{r}} \beta^{3}(t-k) \\ y_{\text {inf }}(t)=\sum_{k=-1}^{M+1} c_{y, k}^{\mathrm{r}} \beta^{3}(t-k),\end{array}\right.$

where $\left(c_{x, k}^{\mathrm{r}}, c_{y, k}^{\mathrm{r}}\right)$ is the coordinate of the control point for the reflected profile spline, which is obtained by a symmetry transform of $\left(c_{x, k}, c_{y, k}\right)$, and where $\left(c_{x,-1}^{\mathrm{r}}, c_{y,-1}^{\mathrm{r}}\right)$ and $\left(c_{x, M+1}^{\mathrm{r}}, c_{y, M+1}^{\mathrm{r}}\right)$ are the coordinates of the phantom control points of the reflected profile. (Note that $c_{0}=c_{0}^{\mathrm{r}}$ and $c_{M}=c_{M}^{\mathrm{r}}$.) We illustrate in Fig. 2 a model of the drop that includes the phantoms at the boundary and the reflection defined by the two edge control points.

\section{Evaluation of the image energy}

\subsection{Image interpolation}

In order to minimize the influence of the discretization, it is suggested to interpolate the image of the drop. B-spline interpolation offers good quality at a limited computational cost. Consequently, cubic-spline basis functions have been used. The interpolating coefficients should first be computed. This can be done using efficient methods [20]. Getting an image value at any position necessitates the evaluation of a limited window of neighboring pixels $(4 \times 4$ for cubic splines $)$.

\subsection{Unified image energy}

\subsubsection{General formulation}

Optimization schemes based on gradient energies are very accurate but their major drawback is their convergence radius. The convergence radius may be increased by first applying a smoothing filter to the image. On one hand, this may result in a loss of details and a decrease in accuracy. On the other hand, statistical region-based image energies provide good convergence, but less accuracy. In order to benefit from the advantages of both methods, a unified image energy has been proposed [19]. This approach uses a new gradient energy that takes into account the gradient direction and that has the advantage of being parameterization-invariant.

Considering a simple surface $S$ with a contour delimited by $C$, the gradient-based image energy is given by

$E_{\text {edge }}=\oint_{C} \mathbf{k} \cdot(\nabla f(r) \times \mathrm{d} r)$,

where $\mathbf{k}$ denotes the unit orthogonal vector to the image plane, and where $\nabla f(r)$ is the gradient of the image $f$ at the point $r$ of the curve. We illustrate in Fig. 3 this integration process, where $\mathbf{n}$ is the inward normal vector.

Using Green's theorem, this can be expressed as the surface integral

$E_{\text {edge }}=\iint_{S} \underbrace{\nabla \cdot \nabla f(\mathbf{s})}_{T_{\mathrm{e}}\{f\}} \mathrm{d} \mathbf{s}$,

where $\nabla \cdot$ is the divergence operator.

The region-based energy discriminates an object from its background by taking in consideration the pixel intensities. It is given by

$E_{\text {region }}=\iint_{S} T_{\mathrm{r}}\{f\}(\mathbf{s}) \mathrm{d} \mathbf{s}$,

where $T_{\mathrm{r}}\{f\}$ is the probability-distribution image.

In order to use region energies, one needs to establish the statistical value of the drop and of the background. Considering that sessile drops are often taken in a dedicated environment under standard lighting conditions, this may be determined only once. If the probability distribution is not known, it may be estimated from a temporary contour during optimization.

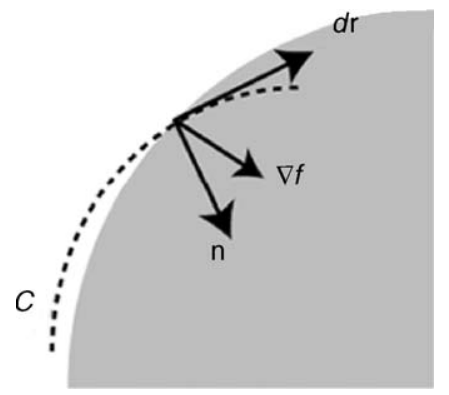

Fig. 3. Gradient and normal to the curve 
Having expressed the gradient energy as a surface integral, the unified image energy may be obtained as

$E_{\text {image }}=\iint_{S} f_{\mathrm{u}}(\mathbf{s}) \mathrm{d} \mathbf{s}$,

where $f_{\mathrm{u}}=\alpha T_{\mathrm{e}}\{f\}+(1-\alpha) T_{\mathrm{r}}\{f\}$. Using Green's theorem again, this unified energy may also be rewritten as the contour integral

$E_{\text {image }}=\oint_{C} f_{\mathrm{u}}^{y}(x, y) \mathrm{d} x=-\oint_{C} f_{\mathrm{u}}^{x}(x, y) \mathrm{d} y$,

where

$f_{\mathrm{u}}^{y}(x, y)=\int_{-\infty}^{x} f_{\mathrm{u}}(x, \tau) \mathrm{d} \tau$

$f_{\mathrm{u}}^{x}(x, y)=\int_{-\infty}^{y} f_{\mathrm{u}}(\tau, y) \mathrm{d} \tau$.

\subsubsection{Spline parameterization of drops}

Let us define $C_{\text {sup }}$ and $C_{\text {inf }}$ so that they represent the nonreflected profile and the reflected profile, respectively, with $C=C_{\text {sup }} \cup C_{\text {inf }}$. The image energy then becomes

$$
\begin{aligned}
E_{\text {image }} & =\int_{C_{\text {sup }}} f_{\mathrm{u}}^{y}(x, y) \mathrm{d} x+\int_{C_{\text {inf }}} f_{\mathrm{u}}^{y}(x, y) \mathrm{d} x \\
& =-\int_{C_{\text {sup }}} f_{\mathrm{u}}^{x}(x, y) \mathrm{d} y-\int_{C_{\text {inf }}} f_{\mathrm{u}}^{x}(x, y) \mathrm{d} y,
\end{aligned}
$$

or, using the parametric representation,

$$
\begin{aligned}
E_{\text {image }}= & \int_{0}^{M} f_{\mathrm{u}}^{y_{\text {sup }}}\left(x_{\text {sup }}, y_{\text {sup }}\right) \frac{\partial x_{\text {sup }}}{\partial t} \mathrm{~d} t \\
& +\int_{0}^{M} f_{\mathrm{u}}^{y_{\text {inf }}}\left(x_{\text {inf }}, y_{\text {inf }}\right) \frac{\partial x_{\text {inf }}}{\partial t} \mathrm{~d} t \\
E_{\text {image }}= & \int_{0}^{M} f_{\mathrm{u}}^{x_{\text {sup }}}\left(x_{\text {sup }}, y_{\text {sup }}\right) \frac{\partial y_{\text {sup }}}{\partial t} \mathrm{~d} t \\
& +\int_{0}^{M} f_{\mathrm{u}}^{x_{\text {inf }}}\left(x_{\text {inf }}, y_{\text {inf }}\right) \frac{\partial y_{\text {inf }}}{\partial t} \mathrm{~d} t .
\end{aligned}
$$

\subsection{Energy derivation}

\subsubsection{Derivation for normal control points}

Using (16) and $\frac{\partial f_{\mathrm{u}}^{x}}{\partial x}=f_{\mathrm{u}}$, the derivative of the image energy may be calculated with respect to the horizontal position of a control point as

$$
\begin{aligned}
\frac{\partial E_{\text {image }}}{\partial c_{x, k}} & =-\oint_{C} f_{\mathrm{u}} \frac{\partial x}{\partial c_{x, k}} \mathrm{~d} y \\
& =-\int_{0}^{M} f_{\mathrm{u}} \frac{\partial x_{\text {sup }}}{\partial c_{x, k}^{\mathrm{r}}} \frac{\partial y_{\text {sup }}}{\partial t} \mathrm{~d} t-\int_{0}^{M} f_{\mathrm{u}} \frac{\partial x_{\text {inf }}}{\partial c_{x, k}^{\mathrm{r}}} \frac{\partial y_{\text {inf }}}{\partial t} \mathrm{~d} t .
\end{aligned}
$$

Note that the computation of $\frac{\partial E_{\text {image }}}{\partial c_{x, k}}$ and of $\frac{\partial E_{\text {image }}}{\partial c_{y, k}}$ can be realized efficiently (see Appendix A).

\subsubsection{Derivation for the axis of symmetry}

The positions of $c_{0}$ and $c_{M}$ are of great importance as they define the position of the whole reflected profile. Furthermore, the position of these points is greatly influencing the contact angle. Consequently, in order to let them adjust to the image energy in the best way, and considering that the symmetry axis is almost horizontal, only their vertical derivative is affected by the symmetry derivative.

The position $\left(x_{\mathrm{h}}, y_{\mathrm{h}}\right)$ is defined in the middle of the control points $c_{0}$ and $c_{M}$. The angle of the axis of symmetry with the horizontal may then be written as

$\tan \theta_{\mathrm{h}}=\frac{c_{y, M}-c_{y, 0}}{c_{x, M}-c_{x, 0}}$.

We can derive the image energy with respect to the position of these boundary control points as follows:

$\frac{\partial E_{\text {image }}}{\partial c_{y, 0}}=\frac{\partial E_{\text {image }}}{\partial y_{\mathrm{h}}} \frac{\partial y_{\mathrm{h}}}{\partial c_{y, 0}}+\frac{\partial E_{\text {image }}}{\partial \theta_{\mathrm{h}}} \frac{\partial \theta_{\mathrm{h}}}{\partial c_{y, 0}}$
$\frac{\partial E_{\text {image }}}{\partial c_{y, M}}=\frac{\partial E_{\text {image }}}{\partial y_{\mathrm{h}}} \frac{\partial y_{\mathrm{h}}}{\partial c_{y, M}}+\frac{\partial E_{\text {image }}}{\partial \theta_{\mathrm{h}}} \frac{\partial \theta_{\mathrm{h}}}{\partial c_{y, M}}$,

where

$\frac{\partial y_{\mathrm{h}}}{\partial c_{y, 0}}=\frac{\partial y_{\mathrm{h}}}{\partial c_{y, M}}=\frac{1}{2}$.

Using (18), we finally get

$\frac{\partial \theta_{\mathrm{h}}}{\partial c_{y, 0}}=-\frac{\partial \theta_{\mathrm{h}}}{\partial c_{y, M}}=\frac{\cos ^{2} \theta_{\mathrm{h}}}{c_{x, 0}-c_{x, M}}$.

The efficient computation of $\frac{\partial E_{\text {image }}}{\partial y_{\mathrm{h}}}$ and of $\frac{\partial E_{\text {image }}}{\partial \theta_{\mathrm{h}}}$ is reported in Appendix B.

\section{B-Snake}

Active contours, or snakes, are widely used in computerassisted tools for segmentation. Some of their applications are medical image analysis or feature tracking in video sequences. Snakes were originally defined as a spline energy minimization under internal and external forces [13]. These forces provide at the same time a way to ensure the smoothness of the curve and a way to adapt to specific features. B-spline snakes (B-snakes) are a particular category of snakes that use a parametric B-spline representation of the curve. While having the same basic philosophy than snakes, they incorporate the smoothness constraint in an implicit fashion. Thus, they provide a very intuitive model, which also requires fewer parameters and is consequently faster. B-snake formulation is further justified by the fact that the optimal solution for a curvature-constrained snake is a cubic spline which may be easily represented using B-spline basis functions [22].

In our implementation, external forces are governing the image energy from Section 3.2. A re-parameterization energy is required for the snake to keep its smoothness (internal energy), which we describe now. 


\subsection{Reparameterization energy}

It has been determined that the number of pixel coordinates taken in consideration in a polynomial-fitting approach is of particular importance [11]. A B-spline is nothing else than a piecewise polynomial, so it is very similar to the polynomial fitting approach for the determination of a contact angle. We can thus expect that the contact angle measured with B-spline will be dependent on the length of the contour taken in consideration. With the phantom boundary conditions, a contact angle depends on the interpolating control point at the edge as well as on the previous control point. Consequently, the control of the distance between knots at the contact points is of particular importance.

A drop with no external forces applied tends to minimize its curvature and takes a spherical shape. It has been shown that the optimal solution for a curvature-constrained snake is a curvilinear cubic-spline snake [22]. In order to increase the curvature of a snake, the distance between knots may be reduced. Considering that a sessile drop under gravity presents an increased curvature near the substrate interface, it is interesting to decrease the distance between knots near the contact points. Finally, in a contact angle measurement application, it is more important to follow closely the contour of the drop at its contact points than at its apex.

Consequently, a progressive (non-uniform) repartition of the control points is constrained and a curve velocity with linear variation is adopted as follows:

$d(t)=\sqrt{\left(x^{\prime}(t)\right)^{2}+\left(y^{\prime}(t)\right)^{2}}=\bar{d}\left(1+\gamma-2 \gamma\left|\frac{2 t}{M}-1\right|\right)$

with $0 \leq \gamma<1$, where $d(t)$ is the norm of the velocity, and where $\bar{d}$ is the average velocity. Note that, if $\gamma=0$, the parameterization is curvilinear.

The internal energy penalizes the deviations of the snake from its ideal parameterization. It is given by

$E_{\text {int }}=\int_{0}^{M}\left(\sqrt{\left(x^{\prime}(t)\right)^{2}+\left(y^{\prime}(t)\right)^{2}}-d(t)\right)^{2} \mathrm{~d} t$

and can be differentiated with respect to the control points position, as in

$$
\begin{aligned}
& \frac{\partial E_{\text {int }}}{\partial c_{x, k}}= \int_{0}^{M} 2\left(1-\frac{d(t)}{\sqrt{\left(x^{\prime}(t)\right)^{2}+\left(y^{\prime}(t)\right)^{2}}}\right) \\
& \times x^{\prime}(t) \mathrm{D} \beta^{3}(t-k) \mathrm{d} t . \\
& \forall \quad 1<k<M-1
\end{aligned}
$$

\subsection{Optimization}

The final snake energy to be minimized is given by

$E=E_{\text {image }}+E_{\text {int }}$.

Following the results from [19], we are using the simple steepest-descent algorithm. In order not to try a too-big step, we normalize them at each iteration. During optimization, we adjust the step length with respect to the variation of displacement from one iteration to the next. Convergence is reached when the step length is below a threshold, and after a prescribed number of iterations during which the contact angles have not evolved (above a certain threshold). The convergence criterion on the contact angle may be adjusted but a typical value is $0.01^{\circ}$. In this way, we achieve a finer control of the convergence of the critical points for the contact angle.

Before actually running the optimization algorithm, an initialization contour is required. This contour may be manually defined placing roughly a few knots or an automatic approach may be adopted. We successfully used simple image size considerations for automatic initialization in a certain number of standard drop images. However, using more advanced initialization approaches could allow producing an initialization scheme of wider applicability. From an initialization spline, the detection of the contour of the drop is realized by a two-step evolution procedure. First, the spline is evolved in a fast-snake mode with a limited number of knots and a variable sampling period to globally detect the drop shape. Then, a finer spline is evolved to accurately detect the contour of the drop. In this optimization step, the number of knots is dynamically adjusted according to the desired knot-spacing at interface and the sampling period is set to the unit pixel. The desired distance between knots at the interface is an important parameter of the algorithm and it is more logical to set this parameter rather than to set the total number of knots.

This simple optimization scheme yields satisfactory results at a reasonable speed (a few seconds).

\section{Implementation and application}

\subsection{Software}

The method described in this article has been programmed as a plugin for ImageJ, which is a free open-source multi-platform Java image-processing program [24]. Our plugin is independent of any imaging hardware and, thanks to ImageJ, any common file format may be used for the drop picture. This plugin is called DropSnake and is made freely available [1].

\subsection{Application examples}

In the following examples, contact angles have been measured using an image energy based solely on the gradient. The relative good contrast of those images did not justify the use of a region component. However, region-based energies are no less attractive and should demonstrate their full power in lowcontrast applications where repeated measurements in similar conditions are performed so that an accurate pixel probability distribution can be obtained.

\subsubsection{Measurement of a contact angle with automatic interface detection}

In order to demonstrate the new possibilities offered by our method, it has been tested on tilted drops as represented on Fig. 4. 


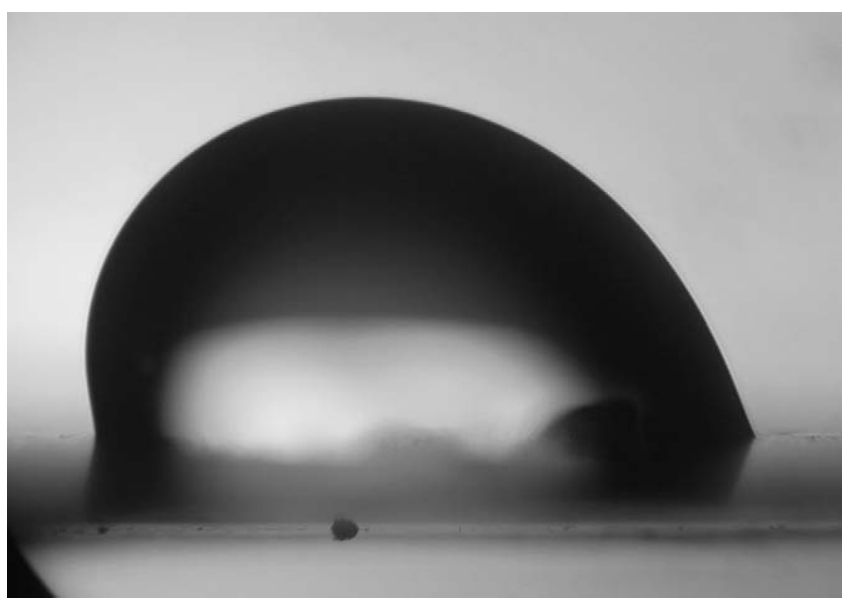

Fig. 4. Drop of ultra-pure water (resistivity $18 \mathrm{Mohms} \mathrm{cm}$, produced by a Millipore MilliQ device, 7-8 $\mu \mathrm{l}$ ) on a vertical PMMA substrate (by Goodfellow, research grad). Picture is courtesy of $\mathrm{M}$. Brugnara, Polymers and Composites Laboratory, University of Trento, Trento, Italy.

In the setup used, the camera is fixed to the sample holder which can be rotated. Due to a small misalignment of the camera horizontal axis with the substrate, the line passing through the contact points reveals a small tilt angle with respect to the horizontal axis of the image. There is a limited drop reflection on the substrate. This image was taken with a digital camera (Nikon995) connected to a $10 \times$ lens. The drop had back-light illumination. After selection of the relevant part of the image, the size of the image was $924 \times 650$ pixel.

The contour of the drop was determined using the following parameters: fast snake with 6 knots after manual initialization, Laplacian smoothing-filter radius of 2.0 pixel, gradientonly image energy, knot-spacing constraint at interface 20 pixel, knot-spacing ratio $2.0(\gamma=1 / 3)$, energy normalization $E_{\text {int }} / E_{\text {image }}=0.3$, convergence criterion $0.01^{\circ}$. The average computation time was $3 \mathrm{~s}$ on a Pentium IV $2 \mathrm{GHz}$.

The detected drop contour is represented in Fig. 5. The measured contact angles for this example are $98.964^{\circ}$ and $66.486^{\circ}$. The detected camera tilt angle was $0.2^{\circ}$.

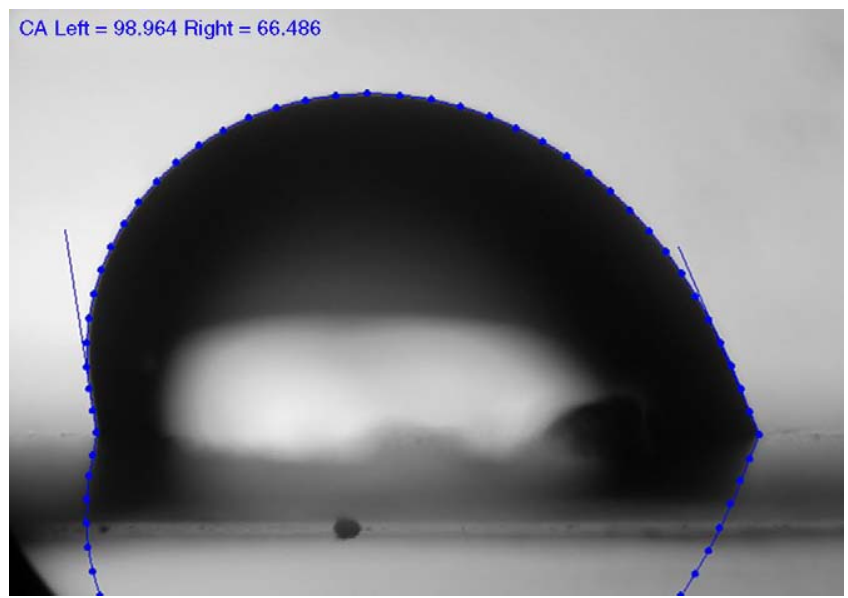

Fig. 5. Detected drop contour and contact angles. The dots represent the position of the knots. The tangents at the contact points are represented by the lines. CA stands for contact angle.

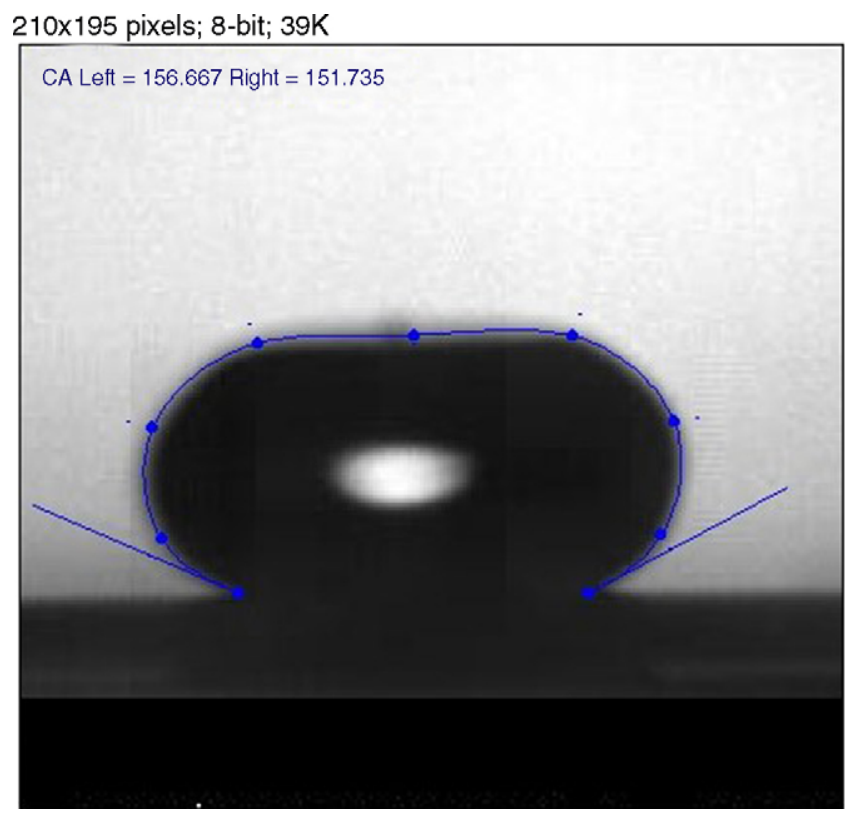

Fig. 6. Advancing angles. The dots represent the positions of the knots. The tangents at the contact points are represented by the lines. CA stands for contact angle.

\subsubsection{Measurement of contact angles for projected drops}

As a second example to illustrate the potential of our method, we show contact angle measurements on projected, and therefore deformed, water droplets. The droplet deformation originates mainly from the inhomogeneous detachment of the droplet from the liquid supply needle by an air flow. The air flow is guided along the needle using a tube that has a diameter slightly larger than the needle. The air flow is launched for $50 \mathrm{~ms}$ by an electric valve operated by a function generator. The pressure in the

\section{0x195 pixels; 8-bit; 39K}

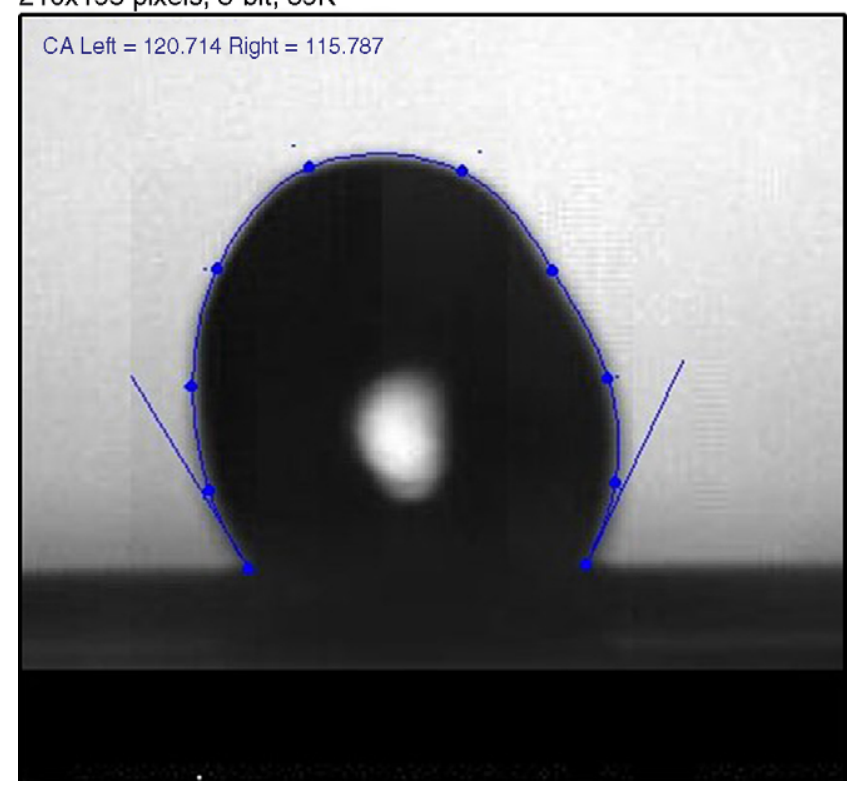

Fig. 7. Receding angles. The dots represent the positions of the knots. The tangents at the contact points are represented by the lines. CA stands for contact angle. 
air tube is adjusted between 80 and 135 mbar, which results in a speed of detached droplet of 0.28 and $0.8 \mathrm{~m} / \mathrm{s}$, respectively. The outer needle diameter is $260 \mu \mathrm{m}$, and the droplet diameter ranges from 1 to $1.4 \mathrm{~mm}$. The flight and the impact of the droplet on the surface was followed by a high-speed camera (Photron Fastcam) at a rate of 10,000 image/s. The droplet shapes are changing during flight, at impact, and bouncing. The droplet edges are then partially out of focus, making them blurry and noisy. The snake-based algorithm allows the exact determination of contact angles from blurred images. The development of dynamic contact angles at surface impact (advancing and receding contact angles, Figs. 6 and 7, respectively) can therefore be studied in detail.

The contour of the drop was determined using the following parameters: fast snake with 5 knots after manual initialization, Laplacian smoothing-filter radius of 2.0 pixel, gradientonly image energy, knot-spacing constraint at interface 20 pixel, knot-spacing ratio $2.0(\gamma=1 / 3)$, energy normalization $E_{\text {int }} / E_{\text {image }}=0.3$, convergence criterion $0.01^{\circ}$.

\subsection{Robustness experiments}

In order to evaluate the robustness of our image-energy and contact angle-measurement approach, various filters have been applied to synthetic drop data. The synthetic data set consisted of three binary images of spherical drops with perfect horizontal symmetry. All the drops had a maximum radius between 300 and 400 pixel. In order to assess the contact angle dependence, each synthetic drop had a different contact angle $\left(29^{\circ}, 81^{\circ}\right.$, and $130^{\circ}$ ).

\subsubsection{Smoothness dependence}

To evaluate the robustness of our method, various Gaussian filters have been applied to the synthetic data set. We have measured the angles of contact on the resulting images and we have compared them to the measurements on the original image. We report the results in Fig. 8.

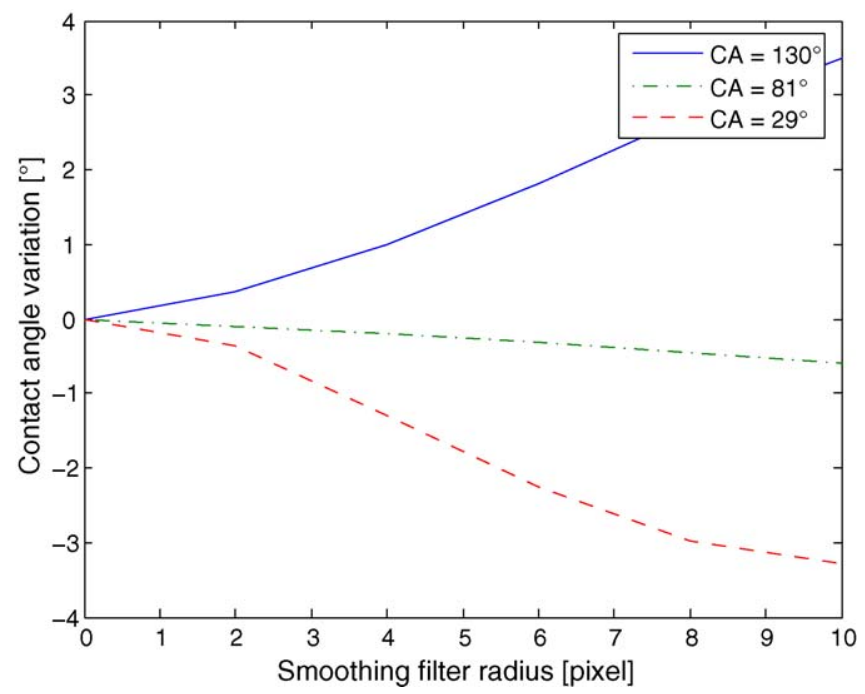

Fig. 8. Measured variation of the contact angles vs. smoothing-filter radius for different initial contact angles.

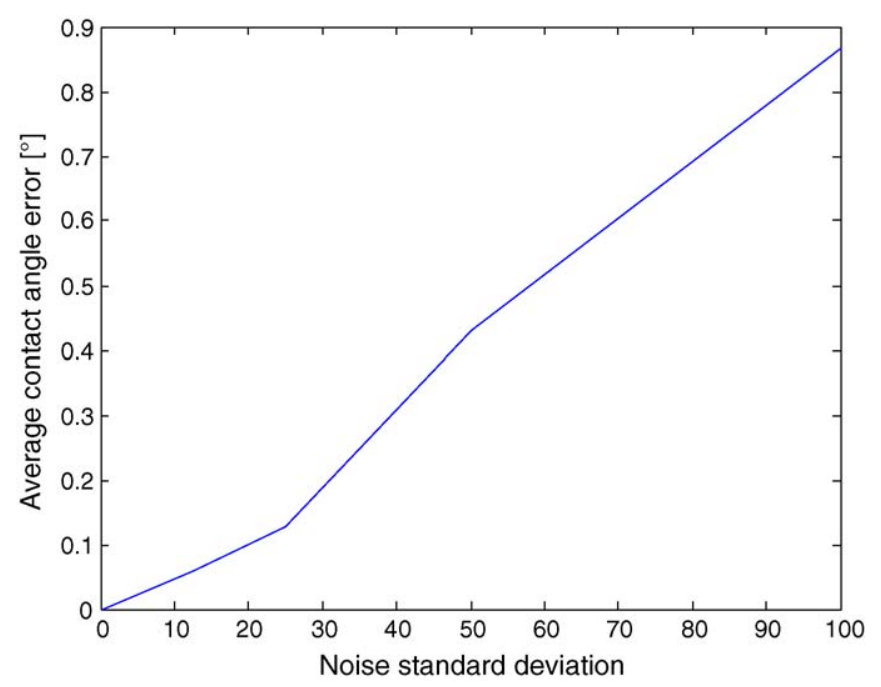

Fig. 9. Contact angle error vs. applied noise.

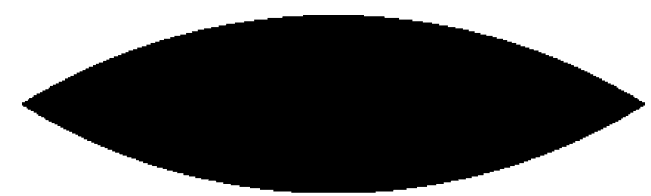

Fig. 10. Image of a synthetic drop with perfect reflection.

Logically, the contact angles near $90^{\circ}$ should not be much affected by the smoothing filter. Angles above $90^{\circ}$ tend to be increased while angles below $90^{\circ}$ tend to be decreased. It should be noted that the measured contact angles remain within a reasonable interval of $\pm 0.36^{\circ}$ for a Gaussian-filter radius of up to 2 pixel.

\subsubsection{Robustness to noise}

Using the same synthetic data, we have assessed the effect of various noise levels on the measured contact angle. Gaussian noises of increasing standard deviation have been applied to the data set. The contact angles have then been measured and compared to their original value. The average contact angle error is represented in Fig. 9.

The contact angle error increases as the noise influence is increased. However, thanks to the robust image energy, it remains reasonable. The average contact angle error is still less than $1^{\circ}$ for applied noise with a standard deviation of up to 100 (pixel span: 8 bit).

For illustration purposes, one image of the synthetic data is represented in Fig. 10. Upon addition of the maximum amount of noise, it corresponds to Fig. 11.

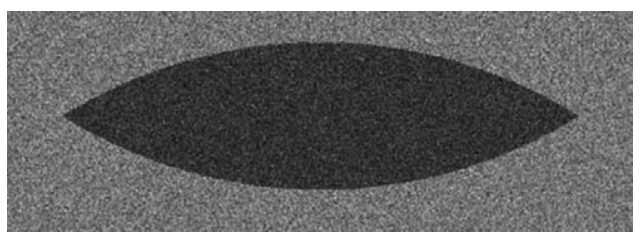

Fig. 11. Image of a synthetic drop with perfect reflection, with an added noise of standard deviation 100 (pixel span: 8 bit). 


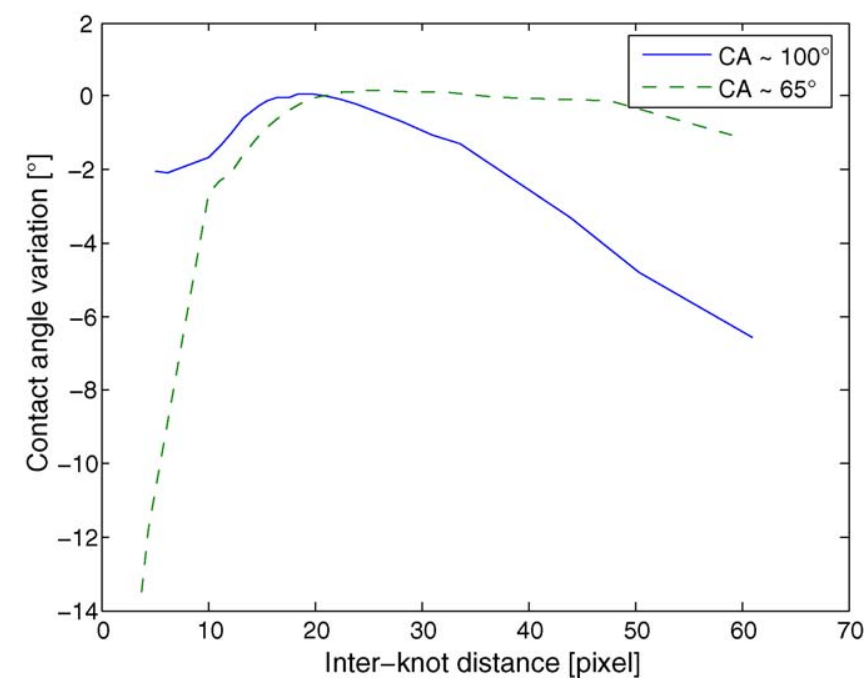

Fig. 12. Contact angle variation vs. inter-knot distance. The contact angle variation is given in reference to the contact angle at 20.9 pixel inter-knot distance.

\subsection{Inter-knot distance}

The contact angle dependence on the distance between knots has been assessed on the drop from Section 5.2.1. Thanks to the variety of contact angles that this drop provides, we can evaluate the influence of the inter-knot distance for angles below and above $90^{\circ}$ on the same image. In order to represent clearly the effect of a lack of control points, the image from Section 5.2.1 has been resized to $231 \times 162$. Indeed, with a smaller resolution, there is less control points for a same inter-knot distance. As the distance between knots may not always be constant around the drop profile, the distance between knots at the contact points is taken into consideration. The contact angle variation with respect to the distance between knots at the contact points is represented on Fig. 12.

Fig. 12, is giving contact angle evolution with progressing inter-knot distance. The contact angles for a 20.9 pixel knot distance have been taken as reference subsequently. At very small inter-knot distance, both contact angles could be visually rejected. Indeed, due to the discrete property of images, considering a too short segment of the contour in the contact angle determination may result in erroneous results (in spite of the sub-pixel interpolation). In addition, due to the limited contrast at the contact points, the contact angle may become uncertain for too small inter-knot distances. This is particularly visible for the small contact angle on Fig. 12. At very large inter-knot distance, we also observe an increased contact angle variability when the knots can no more represent correctly the drop contour. For an inter-knot spacing of about 60 pixel, there was only 5 knots to define the drop contour at this resolution. The maximum limit for the inter-knot distance is related to the minimum number of knots required to accurately represent a drop contour. This minimum number of knots is independent of the resolution but depends on the total curvature of the drop. Thus, the maximum limit on the inter-knot distance may be more easily reached at limited resolution or large contact angles. We see indeed that at very high inter-knot distances both contact angles decrease, but the curve corresponding to the largest contact angle is decreasing much faster.

With reasonable inter-knot distances, a limited dependence of contact angles on inter-knot distance is observed. As a consequence, it is important to keep this parameter fixed for all measurements within a study. The inter-knot distance should be set above a minimum value allowing a reasonable pixel averaging. However, it should allow a sufficient number of knots in order to correctly follow the drop contour. The latter point represents normally no problem and is easy to ensure (10 knots are normally enough). We typically use an inter-knot distance of 20 pixel in our studies.

\subsection{Software evaluation}

Comparison between contact angle measurements methods is a challenging task. Indeed, different methods make different assumptions (e.g., axisymmetricity), necessitates different inputs (e.g., automatic or manual positioning of the substrate level) and can give extra informations (e.g., capillary constant with ADSA). In addition, results can be highly dependent on a particular software application and a same drop model can be used with several edge detection methods sometimes. However, in order to give an evaluation of the implementation of the method, comparisons have been performed with other methods for which the software implementation was available to us. Thus, this subsection cannot claim to compare methods but only software applications based on different methods. In this subsection, we refer to axisymmetric for our implementation of an axis-symmetric method based on Laplace equation. Our axisymmetric implementation was done as a plugin for ImageJ. It is largely based on [10,9] but uses a segmentation method depending on thresholded gradient. This latter point results in a slight overestimation of the contour and of the contact angle. The measurements based on the polynomial method were performed with a commercial software (Windrop++ V4.10, GBX, Romans-sur-Isère, France).

Dynamic contact angles were measured on the same sequence of a drop of water on an isotropic silicium substrate. Images were acquired on a commercial contact angle meter (Digidrop, GBX, Romans-sur-Isère, France). For Dropsnake and the axisymmetric method, the initialization on each frame but the first was using the solution of the preceding frame. Results are presented on Fig. 13.

Considering the offset of our axisymmetric implementation, absolute contact angle considerations may difficultly be taken into account. However, the stability of the measurements may be evaluated. The DropSnake curve is relatively smooth and its measurements are much more stable than measurements from the other methods. In the area of receding angle (frame 270-320) standard deviations were measured to be $0.2^{\circ}, 0.6^{\circ}$ and $1.3^{\circ}$, respectively for DropSnake, the axisymmetric and the polynomial method. It should be noted as well that according to DropSnake and the polynomial method, the drop is not perfectly axisymmetric although it was supposed to be. This latter aspect appears redundantly in our studies as isotropicity is hard to achieve during surface preparation and contact angle measurement. 


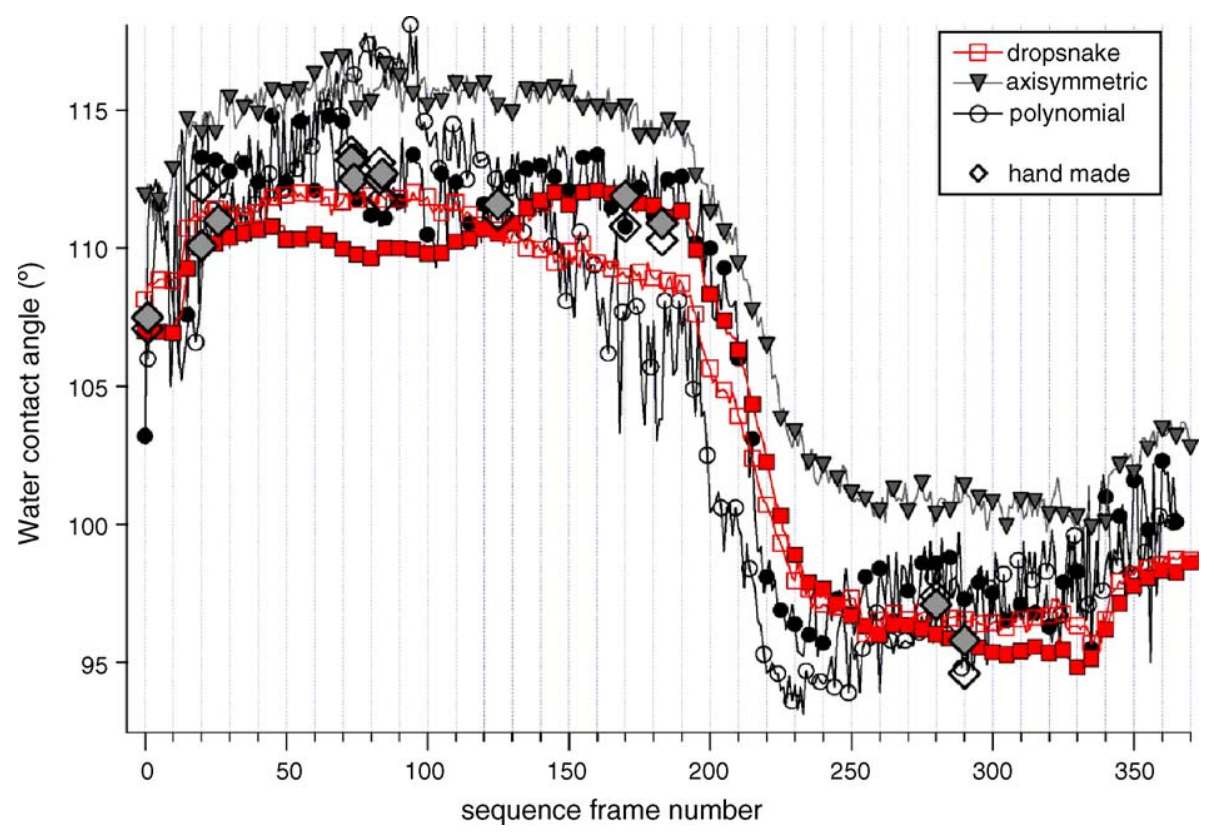

Fig. 13. Comparison of contact angle measurement methods on a sequence of images (370 images). (a) Snake method using our implementation on ImageJ, the DropSnake plugin presented in this paper, 370 measures of the left angle (symbol $\mathbf{\square}$ ) and right angle (symbol $\square$ ). (b) Axisymmetric method using our implementation on ImageJ: 370 measures of one angle (symbol $\mathbf{\nabla}$ ). (c) Polynomial method using a commercial software (GBX Windrop++ V4.10): 370 measures of the left angle (symbol ๑) and right angle (symbol $\bigcirc$ ). (d) Hand-made method: 10 measures of the left angle (symbol $\downarrow$ ) and right angle (symbol $\diamond)$. For visual simplification, symbols are represented every 5 samples.

\subsection{Experiments conclusion}

Using the method described in this article, the contour of the drop as well as the interface (vertical level and tilt) may be automatically detected. A limited presence of drop reflection is sufficient to ensure accurate interface detection. Strongly nonaxisymmetric drops and non-spherical drops may be analyzed successfully.

The method proved to be very robust and little dependency on parameters has been observed (with the exception of the interknot distance discussed in Section 5.4). It presented a limited contact angle dependence when smoothing filters were applied. However, this is already a great improvement compared to methods based on the discretization of the contour of the drop since they fail when confronted to smooth images. Our method also presented excellent inherent robustness to noise. Thus, these good properties allow the method to be used with a very broad range of image types. The inter-knot distance is an important parameter influencing the contact angle results. As a consequence, it is of particular importance to keep this parameter at a reasonable value and constant within a study.

\section{Conclusion}

A new image energy function based on statistical region and gradient information has been applied to the domain of drop shape analysis. This energy function, combined with cubicspline image interpolation, provides a framework for the very robust and highly accurate detection of the contour of the drop, over a broad range of images. The first results obtained with this approach are already promising and the applicability surpasses methods based on a discretization of the contour. The snakebased approach is a novel basis for measuring contact angles of general drops. As it does not make limiting assumptions on the shape of the drop, it may be applied to drops that do not follow any global model. This allows the method to be suitable to a very wide range of applications (e.g. non-axisymmetric drops, tilted drops, projected drops). It is based on a cubic B-spline snake whose minimum-curvature property allows a good description of drop contours with a limited number of control points. In essence, it is similar to the polynomial-fitting approach, but, due to its elasticity, the snake also takes advantage of the global shape of the drop. Thanks to its unifying approach, the snake methodology offers the best tradeoff between the use of the general drop shape to guide the detection of the contour of the drop, and the use of an algorithm with local behaviour to compute contact angles. As a consequence of the definition of a symmetric spline model of the drop, we were able to detect the offset and tilt angle of the line of contact points.

\section{Acknowledgements}

We would like to thank Philippe Thévenaz for the editorial advice.

\section{Appendix A. Derivative computation for normal control points}

Supposing $\theta_{\mathbf{h}}$ small, the following approximation may be done:

$\frac{\partial x_{\mathrm{inf}}}{\partial c_{x, k}}=\frac{\partial x_{\mathrm{inf}}}{\partial c_{x, k}^{r}}, \quad \frac{\partial y_{\mathrm{inf}}}{\partial c_{y, k}}=-\frac{\partial y_{\mathrm{inf}}}{\partial c_{y, k}^{r}}$. 
The energy derivative (17) may be rewritten as:

$$
\begin{aligned}
\frac{\partial E_{\text {image }}}{\partial c_{x, k}}= & -\sum_{l=-1}^{M+1}\left[c_{y, l} \int_{0}^{M} f_{u} \frac{\partial x_{\text {sup }}}{\partial c_{x, k}} D \beta^{3}(t-l) \mathrm{d} t\right. \\
& \left.-c_{y, l}^{r} \int_{0}^{M} f_{u} \frac{\partial x_{\text {inf }}}{\partial c_{x, k}^{r}} D \beta^{3}(t-l) \mathrm{d} t\right]
\end{aligned}
$$

Note that:

$\frac{\partial x_{\text {sup }}}{\partial c_{x, k}}=\frac{\partial y_{\text {sup }}}{\partial c_{y, k}}=\beta^{3}(t-k) \quad \forall \quad 2 \leq k \leq M-2$

and due to the phantom edge definition:

$$
\begin{aligned}
& \frac{\partial x_{\text {sup }}}{\partial c_{x, 1}}=\frac{\partial y_{\text {sup }}}{\partial c_{y, 1}}=\beta^{3}(t-1)-\beta^{3}(t+1) \\
& \frac{\partial x_{\text {sup }}}{\partial c_{x, 0}}=\frac{\partial y_{\text {sup }}}{\partial c_{y, 0}}=\beta^{3}(t)+2 \beta^{3}(t+1) \\
& \frac{\partial x_{\text {sup }}}{\partial c_{x, M-1}}=\frac{\partial y_{\text {sup }}}{\partial c_{y, M-1}}=\beta^{3}(t-(M-1)) \\
& -\beta^{3}(t-(M+1)) \\
& \frac{\partial x_{\text {sup }}}{\partial c_{x, M}}=\frac{\partial y_{\text {sup }}}{\partial c_{y, M}}=\beta^{3}(t-M)+2 \beta^{3}(t-(M+1))
\end{aligned}
$$

The spline representing the reflected contour has identical derivatives for its respective control points.

Note that the cubic spline basis function $\beta^{3}$ as well as its derivative $D \beta^{3}$ have a finite support and the integral in (A.1) does not need to be computed over its whole range. However, care should be taken with the boundaries.

Note also that the integral (A.1) may be calculated as a finite sum. Although the phantom border conditions are complicating the notation, the spline basis function product may be precalculated. These computation considerations were thoroughly investigated in [19], and the phantoms border conditions are only making things (and notation) a little bit more complicated.

Similarly, using (15), the $y$-axis derivative may be obtained:

$$
\begin{aligned}
\frac{\partial E_{\text {image }}}{\partial c_{y, k}}= & \sum_{l=-1}^{M+1}\left[c_{x, l} \int_{0}^{M} f_{u} \frac{\partial y_{\text {sup }}}{\partial c_{x, k}} D \beta^{3}(t-l) \mathrm{d} t\right. \\
& \left.-c_{x, l}^{r} \int_{0}^{M} f_{u} \frac{\partial y_{\text {inf }}}{\partial c_{x, k}^{r}} D \beta^{3}(t-l) \mathrm{d} t\right]
\end{aligned}
$$

\section{Appendix B. Derivative computation for the axis of symmetry}

Here again, it is supposed that $\theta_{\mathrm{h}}$ is small: $\frac{\partial y_{\text {inf }}}{\partial y_{\mathrm{h}}}=2$ and $\frac{\partial x_{\text {inf }}}{\partial y_{\mathrm{h}}}=0$

Hence:

$$
\begin{aligned}
\frac{\partial E_{\text {image }}}{\partial y_{\mathrm{h}}} & =\int_{0}^{M} \frac{\partial f_{u}^{y}}{\partial y} \frac{\partial y_{\text {inf }}}{\partial y_{\mathrm{h}}} \frac{\partial x_{\text {inf }}}{\partial t} \mathrm{~d} t \\
& =2 \sum_{l=-1}^{M+1} c_{x, l}^{r} \int_{0}^{M} f_{u} D \beta^{3}(t-l) \mathrm{d} t
\end{aligned}
$$

The image derivative energy with respect to the angle $\theta_{\mathrm{h}}$ may be written as an average of its computations using $f_{u}^{y}$ and $f_{u}^{x}$ :

$$
\begin{aligned}
\frac{\partial E_{\text {image }}}{\partial \theta_{\mathrm{h}}}= & \frac{1}{2} \int_{0}^{M}\left(\frac{\partial f_{u}^{y}}{\partial y} \frac{\partial y_{\text {inf }}}{\partial \theta_{\mathrm{h}}}+\frac{\partial f_{u}^{y}}{\partial x} \frac{\partial x_{\text {inf }}}{\partial \theta_{\mathrm{h}}}\right) \frac{\partial x_{\text {inf }}}{\partial t} \mathrm{~d} t \\
& +\frac{1}{2} \int_{0}^{M}\left(\frac{\partial f_{u}^{x}}{\partial x} \frac{\partial x_{\text {inf }}}{\partial \theta_{\mathrm{h}}}+\frac{\partial f_{u}^{x}}{\partial y} \frac{\partial y_{\text {inf }}}{\partial \theta_{\mathrm{h}}}\right) \frac{\partial y_{\text {inf }}}{\partial t} \mathrm{~d} t
\end{aligned}
$$

Which can be rearranged as:

$$
\begin{aligned}
\frac{\partial E_{\text {image }}}{\partial \theta_{\mathrm{h}}}= & \frac{1}{2} \int_{0}^{M}\left[\left(\frac{\partial f_{u}^{y}}{\partial y} \frac{\partial x_{\text {inf }}}{\partial t}+\frac{\partial f_{u}^{x}}{\partial y} \frac{\partial y_{\text {inf }}}{\partial t}\right) \frac{\partial y_{\text {inf }}}{\partial \theta_{\mathrm{h}}}\right. \\
& \left.+\left(\frac{\partial f_{u}^{y}}{\partial x} \frac{\partial x_{\text {inf }}}{\partial t}+\frac{\partial f_{u}^{x}}{\partial x} \frac{\partial y_{\text {inf }}}{\partial t}\right) \frac{\partial x_{\text {inf }}}{\partial \theta_{\mathrm{h}}}\right] \mathrm{d} t
\end{aligned}
$$

The terms $\frac{\partial f_{u}^{y}}{\partial x}$ and $\frac{\partial f_{u}^{x}}{\partial y}$ may be computed, however that would require twice the computation over the whole image. In practice, if we are close enough to the drop, the path should remain parallel to the contour and its energy image. That means that the product of the differentiation of the energy image in a direction and the displacement in the same direction should remain small. Hence, we neglect the products $\frac{\partial f_{u}^{x}}{\partial y} \frac{\partial y_{\text {inf }}}{\partial t}$ and $\frac{\partial f_{u}^{y}}{\partial x} \frac{\partial x_{\text {inf }}}{\partial t}$.

The computed derivative is then:

$$
\frac{\partial E_{\text {image }}}{\partial \theta_{\mathrm{h}}}=\frac{1}{2} \int_{0}^{M}\left[\frac{\partial f_{u}^{y}}{\partial y} \frac{\partial x_{\mathrm{inf}}}{\partial t} \frac{\partial y_{\mathrm{inf}}}{\partial \theta_{\mathrm{h}}}+\frac{\partial f_{u}^{x}}{\partial x} \frac{\partial y_{\mathrm{inf}}}{\partial t} \frac{\partial x_{\mathrm{inf}}}{\partial \theta_{\mathrm{h}}}\right] \mathrm{d} t
$$

\section{References}

[1] A.F. Stalder, DropSnake, Biomedical Imaging Group, EPFL, [ON LINE] visited 2005. http://bigwww.epfl.ch/demo/dropanalysis.

[2] S. Herminghaus, Wetting: introductory note, J. Phys.: Condens. Matter. 17 (2005) S261-S264.

[3] T. Young, An essay on the cohesion of fluids, Philos. Trans. R. Soc. Lond. 95 (1905) 65-87.

[4] W. Barthlott, C. Neinhuis, The purity of sacred lotus or escape from contamination in biological surfaces, Planta 202 (1997) 1-8.

[5] Y.T. Cheng, D.E. Rodak, Is the lotus leaf superhydrophobic?, Appl. Phys. Lett. 86 (2005) 144101.

[6] T. Michel, U. Mock, I. Roisman, J. Rühe, C. Tropea, The hydrodynamics of drop impact onto chemically structured surfaces, J. Phys.: Condens. Matter. 17 (2005) S607-S622.

[7] U. Mock, T. Michel, C. Tropea, I. Roisman, J. Rühe, Drop impact on chemically structured arrays, J. Phys.: Condens. Matter. 17 (2005) S595S605.

[8] P.G. de Gennes, Wetting: statics and dynamics, Rev. Mod. Phys. 57 (1985) 827-863.

[9] Y. Rotenberg, L. Boruvka, A.W. Neumann, Determination of surface tension and contact angle from the shapes of axisymmetric fluid interfaces, J. Colloid Interface Sci. 93 (1983) 169-183.

[10] M. Hoorfar, A.W. Neumann, Axisymmetric drop shape analysis (adsa) for the determination of surface tension and contact angle, J. Adhesion 80 (2004) 727-747.

[11] A. Bateni, S.S. Susnar, A. Amirfazli, A.W. Neumann, A high-accuracy polynomial fitting approach to determine contact angles, Colloids Surf. A 219 (2003) 215-231.

[12] O.I. del Rio, D.Y. Kwok, R. Wu, J.M. Alvarez, A.W. Neumann, Contact angle measurement by axisymmetric drop shape analysis and a automated polynomial fit program, Colloids Surf. A 143 (1998) 197-210. 
[13] M. Kass, A. Witkin, D. Terzopoulos, Snakes: Active contour models, Int. J. Comput. Vis. (1988) 321-331.

[14] P. Cheng, D. Li, L. Boruvka, Y. Rotenberg, A.W. Neumann, Automation of axisymmetric drop shape analysis for measurement of interfacial tensions and contact angles, Colloids Surf. A 43 (1990) 151-167.

[15] C. Atae-Allah, M. Cabrerizo-Vílchez, J.F. Gómez-Lopera, J.A. HolgadoTerriza, R. Román-Roldán, P.L. Luque-Escamilla, Measurement of surface tension and contact angle using entropic edge detection, Meas. Sci. Technol. 12 (2001) 288-298.

[16] M.G. Cabezas, A. Bateni, J.M. Montanero, A.W. Neumann, A new dropshape methodology for surface tension measurement, Appl. Surf. Sci. 238 (2004) 480-484.

[17] M.G. Cabezas, A. Bateni, J.M. Montanero, A.W. Neumann, A new method of image processing in the analysis of axisymmetric drop shapes, Colloids Surf. A 255 (2005) 193-200.
[18] H.W. Park, T. Schoepflin, Y. Kim, Active contour model with gradient directional information: directional snake, IEEE Trans. Circuits Syst. Vid. 11 (2001) 252-256.

[19] M. Jacob, T. Blu, M. Unser, Efficient energies and algorithms for parametric snakes, IEEE Trans. Image Process. 13 (2004) 1231-1244.

[20] M. Unser, Splines: a perfect fit for signal and image processing, IEEE Signal Process. Mag. 16 (1999) 22-38.

[21] A. Blake, M. Isard, Active contours, Springer Verlag, London, 1998.

[22] P. Brigger, J. Hoeg, M. Unser, B-Spline snakes: A flexible tool for parametric contour detection, IEEE Trans. Image Process. 9 (2000) 14841496.

[23] R. Bartels, J. Beatty, B. Barsky, An introduction to splines for use in computer graphics and geometric modeling, Morgan Kaufmann Publishers, Los Altos California, 1987.

[24] ImageJ, [ON LINE] visited 2005. http://rsb.info.nih.gov/ij/. 